\title{
Mechanochemical Grinding of Inorganic Oxides ${ }^{\dagger}$
}

\author{
G.R. Karagedov, N.Z. Lyakhov \\ Institute of Solid State Chemistry and \\ Mechanochemistry Russia*
}

\begin{abstract}
A great deal of experimental data on the mechanochemical treatment of inorganic oxides and mixtures thereoffalls into the simple scheme involving the concurrent manifestation of grinding, particle aggregation, and primary crystallite coalescence, respectively. The properties of resulting powders proved to be determined by the position of dynamic equilibrium among the processes taking place. Exerting an influence on the course of one or several processes makes it possible to shift the position of equilibrium and thus to obtain powders with different extents of aggregation, different particle sizes and size distribution curves, or to carry out the treatment under conditions favorable for mechanochemical synthesis. As a result, a weakly agglomerated 15-nm $\alpha-\mathrm{Al}_{2} \mathrm{O}_{3}$ powder was prepared in one case, and either yttrium or calcium-stabilized zirconia formed directly in the mill in the other case.
\end{abstract}

\section{Introduction}

The progress in modern material science is mostly determined by the achievements made in science and the technology of super fine powder production. This evidence is mostly explained by the more stringent requirements imposed on powder substances in the submicron range on the one hand, and by the aroused interest in nanosized powders on the other hand. The development of nanostructured threedimensional materials with unique parameters [1-5] is impossible without mastering the technology for producing weakly agglomerated powders with the mean size of 10-50 nm. However, the use of powders with particles of average size 10-100 nm instead of more coarsely dispersed powders offers rather promising and unexpected possibilities such as the coprocessing of ceramics with metals or the considerable reduction of energy costs due to low-temperature sintering [6].

All these factors brought about the rapid development of ultrafine powder production methods. Among them, the most successful results were obtained by the gas-phase method [7]. From our viewpoint, the method of ultrafine powder production by grinding

\footnotetext{
* Siberian Branch Russian Academy of Science Kutateladze 18, Novosibirsk, 630128, Russia

+ Accepted: June, 2003
}

has largely escaped current attention. However, the mechanochemical grinding of oxides, for example, takes clear superiority over the other methods of fine powder production (above all during the mechanical treatment of the particles in the submicron range, the physical and chemical processes proceed simultaneously, in coordination, and to an inter-correlated pattern). The grinding method is (i) inexpensive, (ii) ecologically clean - since it does not require large volumes of solutions - and (iii) produces particles of the desired size [8]. It should be noted, however, that the oversimplified idea about the direct correlation between the average particle size and the intensity and/or duration of grinding that is valid for coarse grinding (i.e. the higher the intensity and duration of grinding, the smaller is the average size of the powder particles) became absolutely unsuitable in the submicron range [9]. The preparation of nanopowders by direct grinding without taking into account the particular characteristics of the nano-particle behavior under intensive mechanical treatment seems impossible.

The goal of the present study is to describe the general process of intensive mechanochemical grinding, as well as to visualize how an understanding of the features of constituent physicochemical processes makes the task of producing nanopowders of inorganic oxides relatively simple. 


\section{Generai Considerations}

Whe present-day view on the processes occurring in w the different types of grinding machines (i.e. ball, vibration, planetary mill, etc.), in which mechanical energy is transferred to the material by the grinding media is described by the following flow chart:

\section{Coarse grinding \\ (decrease of the particle size) \\ $\downarrow$}

Fine grinding

(further decrease of the particle size and aggregation of particles)

$\downarrow$

Densification of the aggregates $[9,10]$ and destruction of their constituent grains or crystallites (aggregate microstructure refinement and particle welding) $\downarrow$

Recrystallization of the primary particles [11-13], grinding of aggregates and recrystallized particles

By increasing the intensity and/or the time of mechanical treatment of the powder, we can consequently transfer the system from one level to another. It should be noted that on the third or fourth level, the system inevitably achieves the dynamic equilibrium state, that is, the comminution and agglomeration or reduction of crystallites in size and their re-crystallization proceed at equal rates.

Under application of the curves plotted in figure 1, it was possible to obtain a qualitative representation of how the concurrent course of different physicochemical processes influences the size and properties of the treated material and, therefore, of how to predict the consequences of the deliberate alteration of experimental parameters. This figure demonstrates the variation of the powder particle size $(R)$ as a function of the parameter (I), which could be described as the value of the treatment period under a fixed input of mechanical energy during a unit of time (intensity), or the intensity value during the fixed time. In any case, the curve mode is the same.

Curve 1 describes the decrease in the mean particle size if the particles do not aggregate. The fact that the energy needed to destroy the particles increases considerably with the decrease of particle size explains why the inclination of the curve tends towards zero with time if the intensity of the process is fixed. If we fix the time and increase the grinding intensity, then after achieving a particular particle size, the energy of brittle fracture exceeds that of its plastic deformation (brittle-viscous transition), and the grinding terminates.

Curve 2 characterizes the process of agglomeration. It demonstrates the evident fact that under the given conditions, the particles of sizes smaller than the critical one are subjected to spontaneous aggregation. In this case, the higher the intensity of the action on the particle collective, the larger in size particles can be subjected to a plastic deformation that promotes aggregation and hence the more particles can be deformed simultaneously and form agglomerates. Curve 2 also tends towards a plateau, because gravitation forces considerably prevail over those of Van der Waals for the large particles and destroy agglomerates. Besides, the large particles and aggregates are preferably subjected to brittle fracture.

The resulting 1-2 curve determines the minimum particle size that could be achieved in the conditions given and points out the inevitability of dynamic equilibrium when the processes of comminution and aggregation counterbalance each other. It is important to note that after achieving the minimum point, a further increase in the intensity of the powder treatment (or increase in the duration of treatment) would only cause an increase of the particle size. Thus, if the system is located to the right of the minimal size, it is often sufficient to decrease the intensity of mechanical treatment in order to decrease the mean particle size. It is worth noting that the powders obtained under conditions when the system is located to the left of the minimum point are weakly aggregated and preferable for dense ceramic processing, whereas to

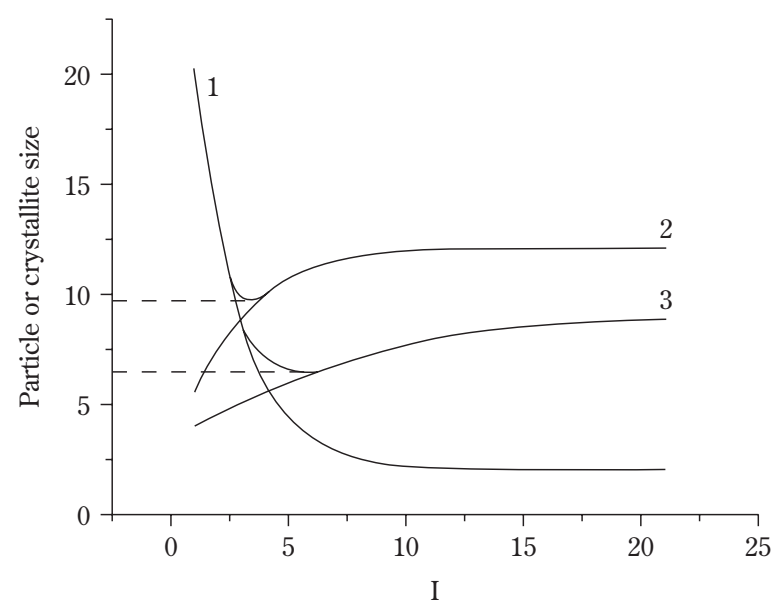

Fig. 1 Schematic representation of particle (crystallite) size versus time (intensity) of mechanical treatment. 
the right of the minimum, the powders are strongly argeregated and can be recommended for ultra-micropoife materials.

The fact that the system achieves the dynamic equilibrium state relative to the formation and decomposition of aggregates does not mean that the other characteristics will not alter. In particular, curve 3 demonstrates the growth of crystallites, whereas the minimum crystallite size is determined by the parabola composed by the curves 1 and 3 . After stabilizing the size of the aggregates, their microstructure refinement can still take place and it terminates later.

It is important to note that the minimum crystallite size is achieved considerably earlier than the dynamic equilibrium state, which is characterized by continuous processes of coalescence and splitting of crystallites. These processes cause a considerable mass exchange between crystallites. Also the areas of local "overheating" with increased reactivity are constantly emerging. In this part of the diagram, the so-called mechanochemical reactions are most intensive. This fact is of the utmost importance for nanogrinding, because on the one hand, it helps to obtain the powder of the required chemical and phase composition, and on the other hand, it intensifies the processes causing contamination of the powder.

In order to achieve a particular goal and to determine the necessary alterations to the experimental conditions, it is of great importance to evaluate exactly what position the system - studied by means of specific equipment - occupies in the diagram shown in Fig. 1. Obviously, the decrease of the powder size to an extent less than the minimum size can only be achieved by displacement of the curves 1,2 , and 3 relative to each other. Thus, curve 1 can be moved to the left and curve 2 to the right by adding surface-active reagents. In this case, it was possible to markedly decrease the minimum possible size. Curve 3 can be shifted to the right (often with simultaneous shifting to the left of the curve 2) by adding an additive that is inert with respect to the powder to be ground and which covers the crystallites, thus preventing their coalescence to aggregates. The subsequent removal of the additives leads to the decomposition of aggregates and to the formation of a powder in the nanometer range.

It should be remembered that one and the same substance behaves in different ways under different modes of intensity or at different stages of mechanical treatment. For example, if organic surfactants are treated with significant intensity, they decompose. Also, minor amounts of water foster the formation of very large aggregates with high bonding strengths (up to the formation of a stone mass) due to "welding" of the hydrated surfaces accompanied by the release of water in accordance with the following quasi-chemical equation:

$$
\text { Bulk-M-OH+OH-M-bulk } \rightarrow \text { bulk-M-O-M-bulk }+\mathrm{H}_{2} \mathrm{O}
$$

Let us illustrate the considerations above by means of specific experimental examples.

\section{Experimental Details}

Commercially available chemically pure alumina, zirconia and magnesia (>99.95\%) were used. Prior to the experiments, the alumina was calcined at various temperatures $\left(1150-1400^{\circ} \mathrm{C}\right)$ until X-ray diffraction patterns detect only the $\alpha$-phase. The mechanical treatment of the powder was performed using an AGO-2 planetary mill. Steel balls of $10 \mathrm{~mm}$ that ranged in effective density from 4 to $8 \mathrm{~g} / \mathrm{cm}^{3}$ were prepared by drilling reach-through holes. The density was then calculated as mass to enveloping sphere volume ratio.

The specific surface area of the powders was measured using the BET method after drying in the flow of argon gas at $200^{\circ} \mathrm{C}$. The X-ray phase analysis and the determination of crystallite size using the line profile analysis was done with the DRON-4 diffractometer supplied with graphite monochromator. The instrumental line broadening as well as the contribution of lattice distortions were taken into account [14]. Electron microscopic studies were performed using the JEM-2000FX2 microscope.

In order to determine the particle size by means of auto-correlation spectroscopy, the powder was subjected to ultrasonic treatment in an aqueous solution of nitric acid $(\mathrm{pH} \sim 4)$, and for the centrifuging process and scanning electron microscopy, in ethanol with 4hydroxybenzoic acid as the dispersant.

\section{Preparation of superfine powders $\left(\alpha-\mathrm{Al}_{2} \mathrm{O}_{3}\right)$}

The grinding of $\alpha-\mathrm{Al}_{2} \mathrm{O}_{3}$ (acceleration 20g) gives evidence of the drastic decrease of the grinding effect with time (see Table 1). The powders treated for 10 and 20 minutes are considerably aggregated because the value of the particle size calculated from the specific surface area equals $\sim 130 \mathrm{~nm}$. This parameter is less significantly than the crystallite size shown in Table 1. From this, it was possible to draw logical deductions that the experimental points are located close to the minima of the curves 1-2 and 1-3 (Fig. 1), 
and that further increase in the duration of mechanicaltreatment will not lead to a further decrease of the wize of the particles and crystallites.

Table 1 Correlation between duration of mechanical treatment and the size of $\mathrm{Al}_{2} \mathrm{O}_{3}$ particles

(20g, balls and crushing cylinders are made of $\mathrm{Al}_{2} \mathrm{O}_{3}$ )

\begin{tabular}{|c|c|c|}
\hline $\begin{array}{c}\text { Duration of grinding } \\
{[\text { min.] }}\end{array}$ & $\begin{array}{c}\text { Crystallite size } \\
{[\mathrm{nm}]}\end{array}$ & $\begin{array}{c}\text { Specific surface area } \\
{\left[\mathrm{m}^{2} / \mathrm{g}\right]}\end{array}$ \\
\hline 0 & 195 & 6.4 \\
\hline 5 & 100 & - \\
\hline 10 & 65 & 12 \\
\hline 20 & 63 & 11 \\
\hline
\end{tabular}

Figure 2 illustrates the change of the crystallite sizes of the alumina, magnesia, and ferric oxide by means of a relatively low-intensity grinding regime where the intensity is insufficient for a visible coalescence of particles and where the clearly detectable minimum is missing.

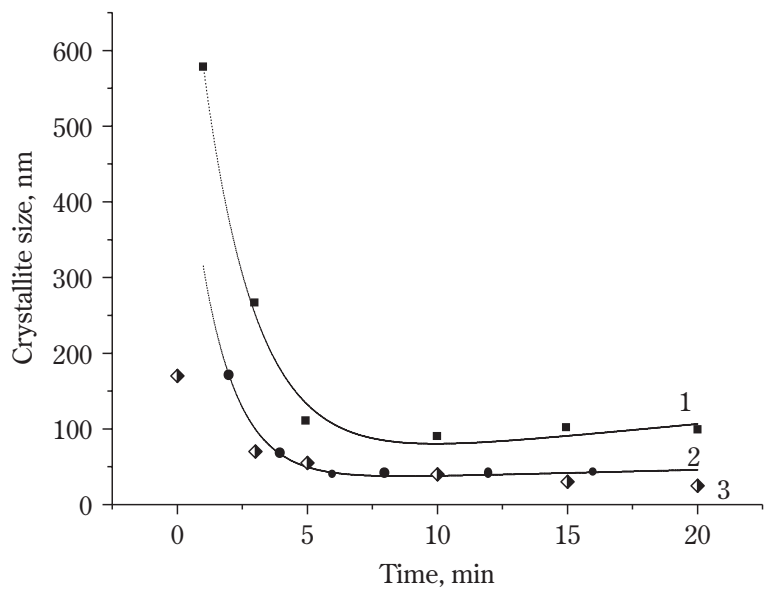

Fig. 2 Crystallite size of $\mathrm{Al}_{2} \mathrm{O}_{3}-1, \mathrm{MgO}-2, \mathrm{Fe}_{2} \mathrm{O}_{3}-3$ as a function of milling time

However, as we increase the intensity of grinding, in the plot showing the dependence of the particle size on the time (Fig. 3), both descending and ascending branches of curve 1-2 could be demonstrated (see Fig. 1). Curves of this type in coordinates "specific surface area - grinding time" could be observed under mechanical activation of numerous inorganic substances [15]. Such evidence is commonly accounted for the competition between the processes of

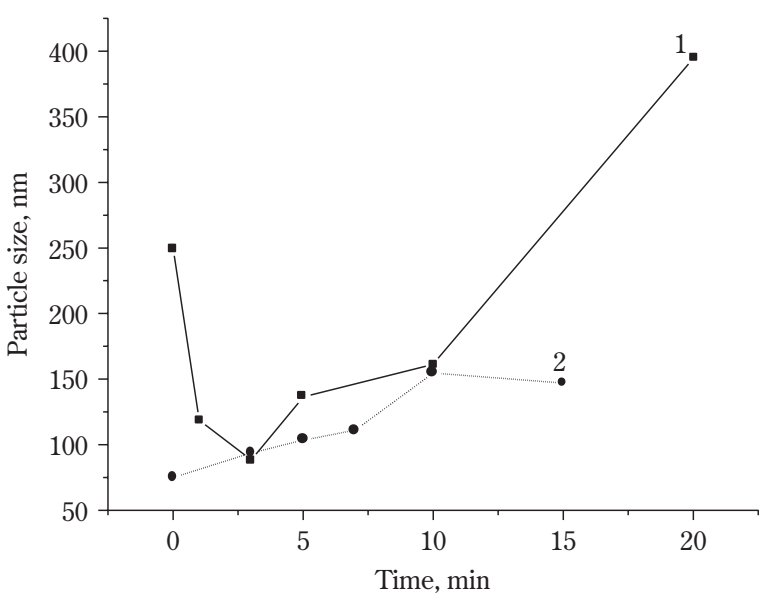

Fig. 3 Particle size of coarse- 1 and fine-2 aluminas calculated from the specific surface area versus time of milling

aggregation and grinding.

As was expected, if the initial powder is already represented by the particles with the mean size close to the minimum, then we can obtain only the ascending part of the curve (curve 2, Figure 3), by means of intensive mechanical treatment.

Obviously, similar curves can be obtained by varying the intensity of the mechanical treatment as well. For example, in Table 2, the influence of the density of the grinding media is shown that is proportional to the kinetic energy of the balls. The dependence of the specific surface area (from our viewpoint, it is exactly this value that reflects the size of the particles and dense aggregates) on the ball density is of an extreme character, which is predicted by the curve 1-2. Although with the increase of ball density, the energy of an impact also increases proportionally, the specific surface area of the powder achieves a particular value and then decreases.

Table 2 Influence of the ball density on the $\alpha-\mathrm{Al}_{2} \mathrm{O}_{3}$ grinding

\begin{tabular}{|l|c|c|c|c|}
\hline $\begin{array}{c}\text { Grinding } \\
\text { time, } \\
20 \mathrm{~g}\end{array}$ & $\begin{array}{c}\text { Material } \\
\text { of } \\
\text { balls }\end{array}$ & $\begin{array}{c}\text { Effective } \\
\text { density } \\
\text { of balls } \\
{\left[\mathrm{g} / \mathrm{cm}^{3}\right]}\end{array}$ & $\begin{array}{c}\text { Specific } \\
\text { surface } \\
\text { area } \\
{\left[\mathrm{m}^{2} / \mathrm{g}\right]}\end{array}$ & $\begin{array}{c}\text { Crystal } \\
\text { size } \\
{[\mathrm{nm}]}\end{array}$ \\
\hline $20 \mathrm{~min}$ & $\mathrm{Al}_{2} \mathrm{O}_{3}$ & 3.9 & 12 & 63 \\
\hline $15 \mathrm{~min}$ & Steel & 3.9 & 11 & 51 \\
\hline $15 \mathrm{~min}$ & Steel & 5.4 & 17.5 & \\
\hline $15 \mathrm{~min}$ & Steel & 8 & 8 & 45 \\
\hline $15 \mathrm{~min}$ & WC & $16-17$ & - & 66 \\
\hline $15 \mathrm{~min}, 5 \% \mathrm{Al}$ & WC & $16-17$ & - & 39.5 \\
\hline
\end{tabular}


Thenbriatieness of tungsten carbide balls brings aloout a greater contamination of the powder because of the associated wear. This leads to an ambiguity in the interpretation of the specific surface area values; hence, the latter was not measured. However, this fact does not interfere with determination of the crystallite size. The curve of type 1-3 becomes evident in this case as well.

It should be noted that the experimental data presented here were specially selected by the authors for the sole purpose of proving the evidence that (i) in order to decrease the powder particle size, it is not always rational to increase either the duration of treatment or the grinding intensity, and (ii) it is impossible to decrease the particle size to a value less than the minimum one without alteration of some other parameters. In practice, particular values obtained under different conditions depend on numerous factors, including those which in turn depend on the powder pre-treatment history (see Table 3). The suggestion that the temperature of alumina calcination effects the toughness through the perfectness of crystal structure seems natural.

Table 3 Effect of $\mathrm{Al}_{2} \mathrm{O}_{3}$ calcination temperature on specific surface area of mechanochemically treated powders subjected to successive acid leaching

\begin{tabular}{|c|c|c|c|c|}
\hline Prehistory & $\begin{array}{c}\text { Treatment } \\
\text { time } \\
{[\mathrm{min}]}\end{array}$ & $\begin{array}{c}\text { Acceleration } \\
{\left[\mathrm{m} / \mathrm{s}^{2}\right]}\end{array}$ & $\begin{array}{c}\text { Ball } \\
\text { diameter } \\
{[\mathrm{mm}]}\end{array}$ & $\begin{array}{c}\text { Specific } \\
\text { surface } \\
{\left[\mathrm{m}^{2} / \mathrm{g}\right]}\end{array}$ \\
\hline $1100^{\circ} \mathrm{C}$ & 20 & 20 & 10 & 28 \\
\hline $1300^{\circ} \mathrm{C}$ & 20 & 20 & 10 & 22 \\
\hline $1450^{\circ} \mathrm{C}$ & 20 & 20 & 10 & 20 \\
\hline & & & & \\
\hline $1100^{\circ} \mathrm{C}$ & 20 & 20 & 5 & 23 \\
\hline $1300^{\circ} \mathrm{C}$ & 20 & 20 & 5 & 19 \\
\hline $1450^{\circ} \mathrm{C}$ & 20 & 20 & 5 & 12 \\
\hline & & & & \\
\hline $1100^{\circ} \mathrm{C}$ & 40 & 40 & 5 & 33 \\
\hline $1300^{\circ} \mathrm{C}$ & 40 & 40 & 5 & 30 \\
\hline $1450^{\circ} \mathrm{C}$ & 40 & 40 & 5 & 27 \\
\hline
\end{tabular}

Treatment by commonly used surfactants that reduce the particle surface energy is not effective within the nanometer range, because they cannot prevent the fusion of crystallites.

On the contrary, the addition of a reagent with some adhesion to the powder in amounts sufficient to cover the surface of the particles may sterically pre- vent crystallites from coalescence, thus reducing the achievable size [16], although the particles can aggregate better. The subsequent removal of the additive by dissolving or evaporation will destroy the aggregates and form a weakly agglomerated powder with very small particle sizes.

The additive should meet rigid and rather contradictory requirements. It should have good adhesion to the powder, but be chemically inert with it. It should be resistant to the high pressure and temperature generated in the area of ball impact; it should be easy to remove after grinding; and it should not be ductile in order to prevent slippage of powder particles relative to each other.

Among inorganic oxides, these requirements are often met by metals and carbon which do not react with many oxides and which can be removed by dissolving in acids or alkalis along with evaporation within the temperature range insufficient for the growth of oxide particles.

In Table 4 (see also the bottom line in Table 2), one can see the sizes of crystallites obtained by grinding $\mathrm{Al}_{2} \mathrm{O}_{3}$ for 15 minutes with the addition of $\mathrm{Al}$ as the most preferable from the viewpoint of purity, $\mathrm{Fe}$ as the metal with the strongest (except Pt) interaction with the alumina surface [17], and $C$ that lacks lubricating properties. As a result, we obtained the mean particle size which is considerably less than that of the crystallites milled without additives (see Fig. 2, Table 2).

The treatment of the product obtained as above with hydrochloric acid removes the metal and, after careful drying, we obtained a weakly agglomerated

Table 4 Crystallite size achieved after grinding for $15 \mathrm{~min}$. with steel balls supplemented with various additives

\begin{tabular}{|c|c|c|c|c|}
\hline Additive & $\begin{array}{c}\text { Acceleration } \\
{\left[\mathrm{m} / \mathrm{s}^{2}\right]}\end{array}$ & $\begin{array}{c}\text { Ball } \\
\text { diameter } \\
{[\mathrm{mm}]}\end{array}$ & $\begin{array}{c}\text { Additive } \\
\text { amount } \\
{[\mathrm{wt} . \%]}\end{array}$ & $\begin{array}{c}\text { Crystallite } \\
\text { size } \\
{[\mathrm{nm}]}\end{array}$ \\
\hline $\mathrm{Al}$ & 40 & 5 & 5 & 19 \\
\hline $\mathrm{Al}$ & 40 & 5 & 15 & 24 \\
\hline $\mathrm{Al}$ & 40 & 5 & 20 & 33 \\
\hline $\mathrm{Al}$ & 40 & 5 & 10 & 32 \\
\hline $\mathrm{Fe}$ & 40 & 10 & 10 & 22 \\
\hline Fe & 40 & 5 & 10 & 26 \\
\hline Acetylene black & 20 & 5 & 10 & 38 \\
\hline Acetylene black & 20 & 5 & 15 & 37 \\
\hline Acetylene black & 20 & 5 & 20 & 34 \\
\hline Acetylene black & & & & \\
\hline
\end{tabular}


powder: For example, in Figure 4, we illustrate the dependence of the mean size of the alumina powder W particles calculated from the specific surface area on the milling time, after removal of the iron.

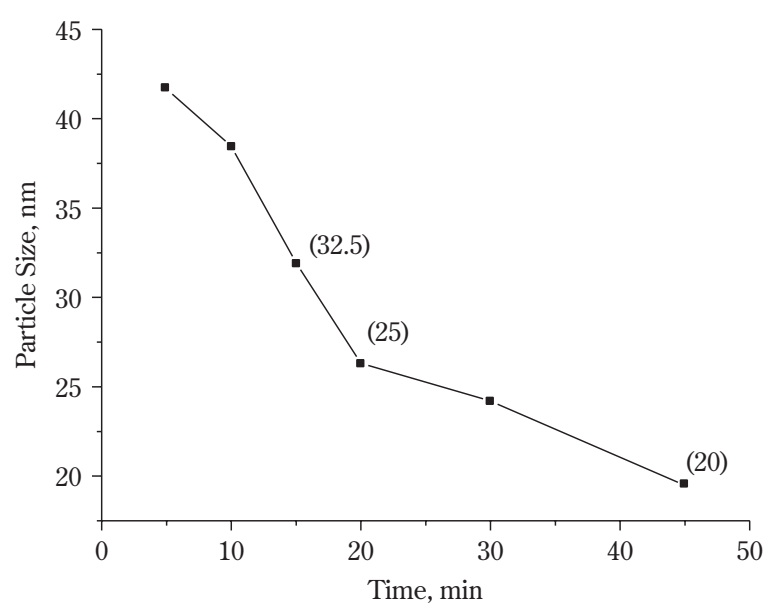

Fig. 4 Particle size of alumina milled with $10 \%$ of $\mathrm{Fe}$ and leached with $\mathrm{HCl}$ as a function of the time of mechanical treatment

The crystallite sizes derived from the X-ray data are given in brackets. For a 25 -nm powder, the particle size was additionally verified by laser autocorrelation spectroscopy, which demonstrates that approximately 80-90 mass\% of the powder truly has this size.

However, electron microscopy images show some large $(100-200 \mathrm{~nm})$ particles, which are presumably the aggregates. The latter could have formed due to an incomplete coverage of the alumina crystallites by iron during the grinding process. If the conditions of mechanical treatment are to be optimized, additional studies are necessary, whereas a mere increase of the ductile metal ( $\mathrm{Fe}, \mathrm{Al}, \mathrm{Zn}, \mathrm{Cr}$ ) content makes the efficiency of grinding deteriorate (Table 4).

In this study, the powder was redispersed in ethanol, treated by ultrasound with addition of 4hydroxybenzoic acid [18]. The resultant suspension was centrifuged for 1 hour at $3000 \mathrm{rpm}$. The coarse ( $\sim 30 \%)$ powder was isolated as the centrifugate. The electron microscopy image of the residual oxide (see Fig. 5) gives evidence of the lack of large particles and the resultant particle size distribution is rather narrow. After drying at $200^{\circ} \mathrm{C}$, the powder had a specific surface area of $107 \mathrm{~m}^{2} / \mathrm{g}$; crystallite size was 16 nm.

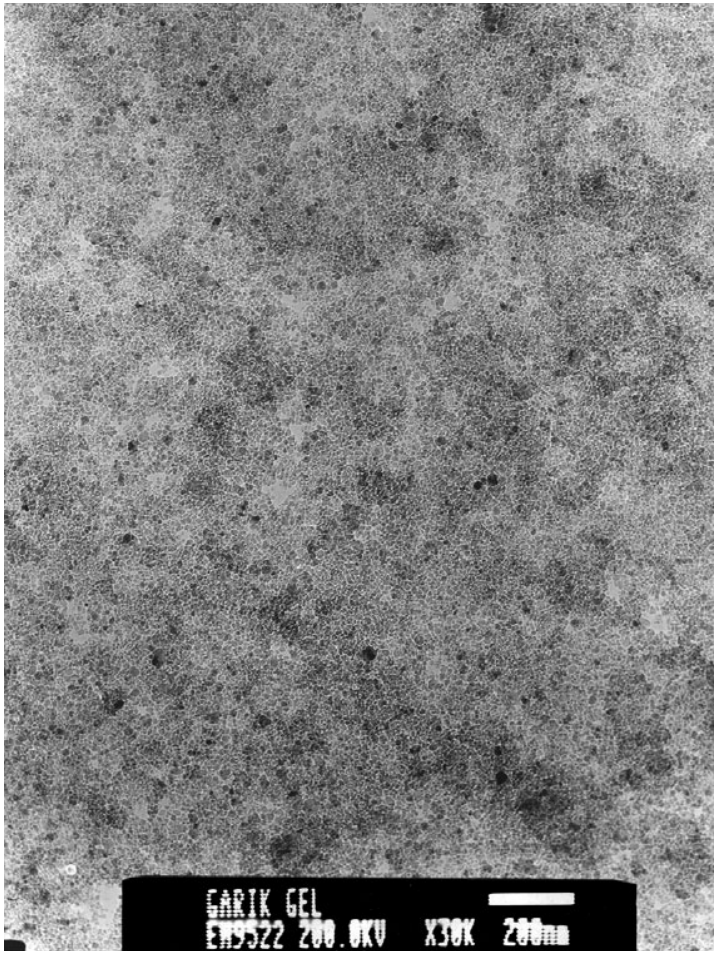

Fig. 5 Electron micrograph of the fines of milled and washed-off alumina

\section{Contamination during Grinding}

In all probability, the most serious disadvantage of using high-energy mills for grinding fine and ultrafine inorganic oxide powders is the inevitable contamination of the powdered material by the wear of the grinding media and, to a lesser extent, of the mill shell. As a first approximation, the level of contamination increases at the ratio $t / r^{2}$, where $t$ is the grinding time, and $\mathrm{r}$ is the crystallite size of the oxide powder. In particular, 10 minutes of grinding $\mathrm{Al}_{2} \mathrm{O}_{3}$ using balls made of $\mathrm{ZrO}_{2}$ at an acceleration of $20 \mathrm{~g}$ are sufficient to show up in the X-ray image as three peaks that correspond to the zirconium oxide. The kinetics of iron accumulation, if a steel shell and steel balls are used, is shown in Figure 6.

Although by optimizing the conditions of the mechanical treatment, in particular by increasing the quality of the grinding media, the wear can be considerably reduced, but the problem of obtaining fine powders cannot be solved by this method.

Previously [19], we already noted that long-term boiling of the $\mathrm{Al}_{2} \mathrm{O}_{3}$ treated by steel balls in hydrochloric acid could not totally eliminate contamination. In the powder treated for $20 \mathrm{~min}$. at an acceler- 


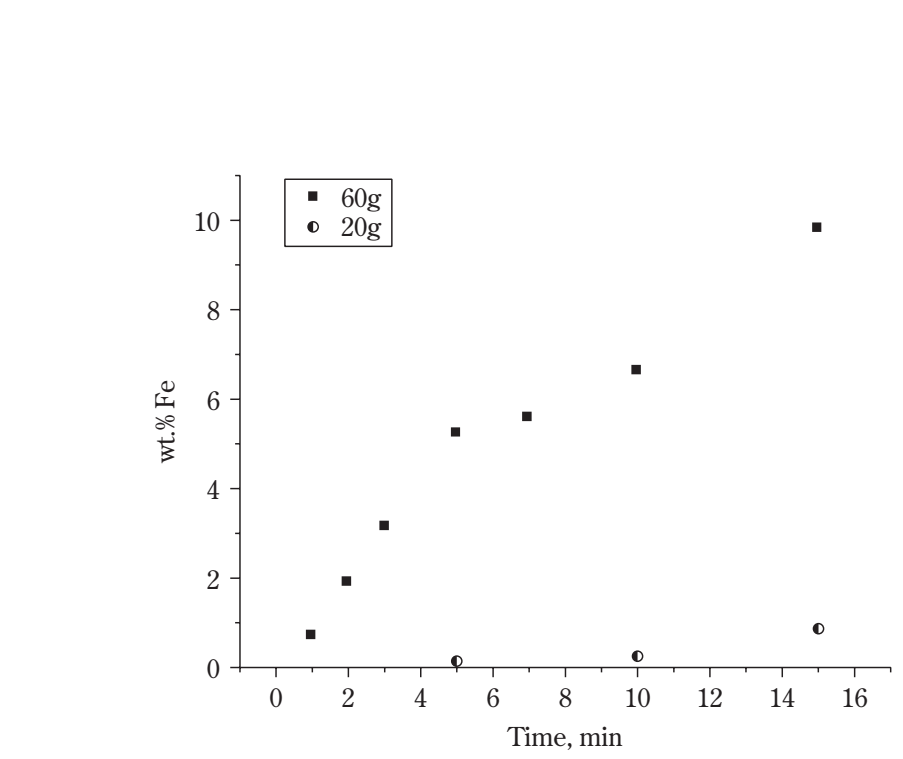

Fig. 6 Percentage of iron contamination as a result of alumina grinding

ation of $40 \mathrm{~g}$, the residual iron was $1.5 \%$, and residual chromium and silicon approximately $0.5 \%$ each. It was noted that Mössbauer spectra identify $\mathrm{Fe}^{3+}$ ions as being surrounded by oxygen.

Additional studies made by ESR spectroscopy have unambiguously supported the assumption on the formation of the $\mathrm{Al}_{2} \mathrm{O}_{3}-\mathrm{Fe}_{2} \mathrm{O}_{3}$ solid solution under intense grinding. The logical conclusion is that the $\alpha-\mathrm{Al}_{2} \mathrm{O}_{3}$ crystal lattice also contains $\mathrm{Cr}$. So, after the intense grinding of alumina with steel balls, when the system falls into the range of plastic deformation (right branch of the curve 1-3 shown in Fig. 1), the following series of mechanochemical reactions takes place:

$$
\begin{aligned}
& \mathrm{Fe}+\mathrm{O}_{2}\left(\mathrm{H}_{2} \mathrm{O}\right) \rightarrow \mathrm{Fe}_{2} \mathrm{O}_{3} \\
& \mathrm{Fe}+\mathrm{Fe}_{2} \mathrm{O}_{3} \rightarrow \mathrm{Fe}_{3} \mathrm{O}_{4}, \mathrm{FeO} \\
& \mathrm{Al}_{2} \mathrm{O}_{3}+\mathrm{Fe}_{3} \mathrm{O}_{4}, \mathrm{FeO} \rightarrow \mathrm{Al}_{2-\mathrm{x}} \mathrm{Fe}_{\mathrm{x}} \mathrm{O}_{3}
\end{aligned}
$$

The first two reactions were proved by special experiments. It was shown that a durable grinding of alumina (or some other oxides) in the sealed shells causes a considerable pressure drop. When the rubber gasket is punctured by the syringe filled with liquid, the liquid penetrates the shell. So, it is natural to suppose that oxygen in the grinding chamber is consumed to produce ferric oxide. Besides this, it was shown that 30 minutes of grinding $\mathrm{Fe}_{2} \mathrm{O}_{3}$ is sufficient for only $\mathrm{FeO}$ responses are being detectable on X-ray.

Since unoxidized metals are not able to react with alumina, we can consider grinding in a dry inert atmosphere as the first step for obtaining fine powders of alumina. In this case, a subsequent treatment by acid would help to obtain an extremely low contamination by iron and chromium residuals.
To this end, a specially constructed mill chamber was evacuated by heating $\left(\sim 200^{\circ} \mathrm{C}\right)$ and then filled with Ar dried over sodium metal up to the pressure of $2 \mathrm{~kg} / \mathrm{cm}^{3}$.

The tests showed that the iron content in the samples subjected to acid leaching underwent an unexpectedly sharp increase. One may surmise in this case that encapsulation of tiny metal particles in the dense oxide aggregates occurs, thus reducing the efficiency of acid treatment. The lack of moisture and, hence, water adsorption layers covering the particles, facilitates the interaction between particles and their caking-up to form dense aggregates.

The data presented in Figure 7 validate this hypothesis. Water layers between the particles decrease the friction and hinder the formation of dense aggregates. This phenomenon could be viewed visually in the form of a significant (immeasurable) rate of the powder lightening (its color changes from black to white) in the process of its washing off in acid.

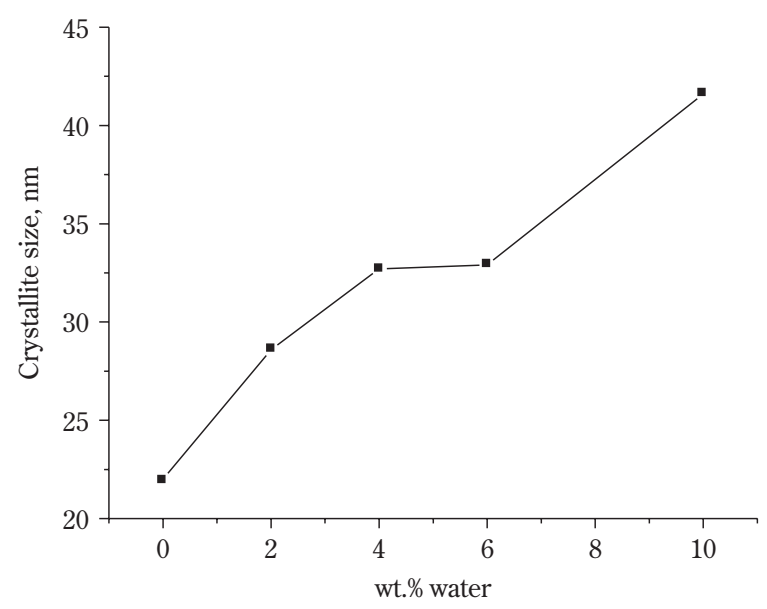

Fig. 7 Crystallite size of ground alumina as a function of the amount of water added

It is obviously impossible to prevent the oxidation of iron by water and, hence, the contamination of $\mathrm{Al}_{2} \mathrm{O}_{3}$ due to mechanochemical reaction with $\mathrm{Fe}_{2} \mathrm{O}_{3}$. But the experiments described above reveal one more mechanism of contamination of the oxide powder, that is, the encapsulation of fine metal particles, if the amount of the additive is insufficient for an effective decomposition of the particles in the aggregates.

The addition of metals may partially compensate the lack of a water layer by acquiring its functions. For example, the addition of $5-10 \%$ of Fe allowed us to obtain an $\alpha-\mathrm{Al}_{2} \mathrm{O}_{3}$ powder with a mean particle size of 
onmand a residual iron content of only $0.02 \%$.

Nemarkably, the starting alumina contained $0.035 \%$

W of iron, so that in this case, we observe the phenomenon of "mechanochemical purification" of the powder. This effect seems rational, especially if we consider the possibility of segregation of the impurity atoms on the surface of the powder. During intense grinding, the surface of the powder multiplies many times. At the same time, the increased diffusion ability of the ions in the particles subjected to intense plastic deformation provides a rapid enrichment of the surface layer by the impurity. The subsequent treatment with an acid dissolves the strongly defective and even amorphous [20] surface layer.

In some cases, it was found promising to use an additive consisting of two substances, one aimed at separating the crystallites from the aggregates, whereas the other has an oxygen affinity and prevents oxidation or even has an ability to mechanochemically reduce the alien metal oxide. The latter reactions are well studied [21-24].

Thus, grinding the alumina with the mixture of $\mathrm{Al}$ and $\mathrm{Fe}$ at ambient atmosphere followed by acid treatment enables the production of powders with a specific surface area of $70 \mathrm{~m}^{2} / \mathrm{g}$, crystallite sizes of 24 $\mathrm{nm}$, and iron residues of less than 0.06 .

\section{Grinding $\mathrm{ZrO}_{2}$}

An intensive mechanical treatment of zirconium dioxide is characterized by a phase transformation $[25,26]$ which is supposed to be due to the contribution of surface energy into the Gibbs potential value. The increase of the specific surface area converts the oxide into the tetragonal or cubic modification and becomes thermodynamically stable. For coarse powders, monoclinic zirconia is known as the stable one.

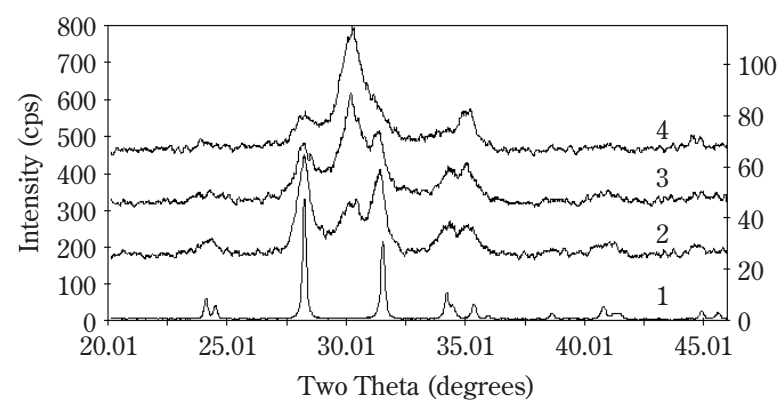

Fig. 8 X-ray patterns of zirconia depending on milling time. 1starting; 2- 5 min.; 3 - 10 min; 4- 15 min.
Figure 8 shows the X-ray patterns of zirconium dioxide powder samples that were initially represented by $100 \%$ monoclinic modification and that were mechanically treated at different periods of time. In Figure 9, one can see the alterations occurring during subsequent thermal annealing of one of the samples. As seen, even a short period of mechanochemical activation under these conditions is sufficient for around $50 \%$ phase transition of the monoclinic modification into a more symmetrical one. This fact was evidenced by the peak at approximately 30 degrees. Thermal treatment of the milled samples initially leads to a more pronounced phase change, but with further increase of temperature, this trend reverses.

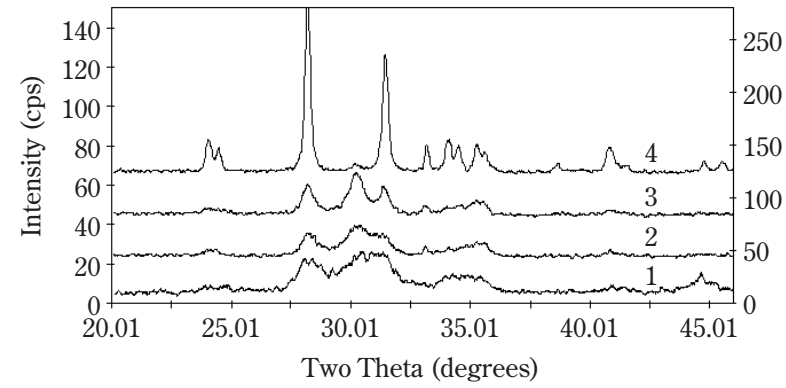

Fig. 9 X-ray pattern of zirconia milled at $40 \mathrm{~g}$ for $10 \mathrm{~min}$. (1) and annealed for 1 hour at $700^{\circ} \mathrm{C}(2), 800^{\circ} \mathrm{C}(3)$ and $900^{\circ} \mathrm{C} \mathrm{(4)}$.

A similar phenomenon was reported in [27], where the data were correlated with the crystallite size, namely that with the increase of crystallite size, the modification became monoclinic and vice versa.

Nevertheless, only the due consideration of the nature and the entirety of processes that take place during intensive mechanochemical grinding may be able to satisfactorily explain the total body of experimental evidence:

- mechanical treatment of the tetragonal $\mathrm{ZrO}_{2}$ modification caused its rapid transformation into a monoclinic one [27];

- mechanical treatment of the monoclinic modification led to its transformation into a tetragonal one. In this case, the extent of transformation after grinding with tungsten carbide balls during a period of as much as 50 hours was only $45 \%$ [25], whereas intensive treatment with steel balls led to a $100 \%$ tetragonal modification after only $\sim 20$ min [26]; 
- thermartreatment of the mechanochemically synde thesized tetragonal modification even at 800${ }^{N} 900^{\circ} \mathrm{C}$ transformed it into a monoclinic one ([25,28], Figure 9), whereas by means of special experiments, we demonstrated that the onset of sintering of the milled zirconium dioxide was detected only at $1000-1050^{\circ} \mathrm{C}$.

All these facts appear to be consistent only with the supposition that an intensive or durable mechanical treatment leads to the condition of a dynamic equilibrium between the processes of crystallite fracture and coalescence (curve 1-3, Figure 1). The crystallites of sizes larger than the threshold size and, hence in a phase of monoclinic modification, divide finely and transform to the tetragonal phase. The opposite process of coalescence of small particles causes a reverse change of phase. The rates of these processes in the equilibrium state are equal, which is why the authors [25] failed to obtain more than $45 \%$ transition, this result corresponds approximately to that shown in Figure 8.

In our opinion, a rather impressive result is given in Figure 10. After 10 minutes of grinding with steel balls at a relatively low intensity $(20 \mathrm{~g})$, it can be seen that the X-ray lines are considerably broader, and tetragonal phase is clearly detectable. However, if this experiment is carried out with the shell cooling water switched off so that the background temperature in the shell increases to $50^{\circ} \mathrm{C}$ as determined in accordance with [29], then the phase transition does not take place and the X-ray lines are notably narrower.

Such significant alterations with only a slight change to the temperature can be explained only by

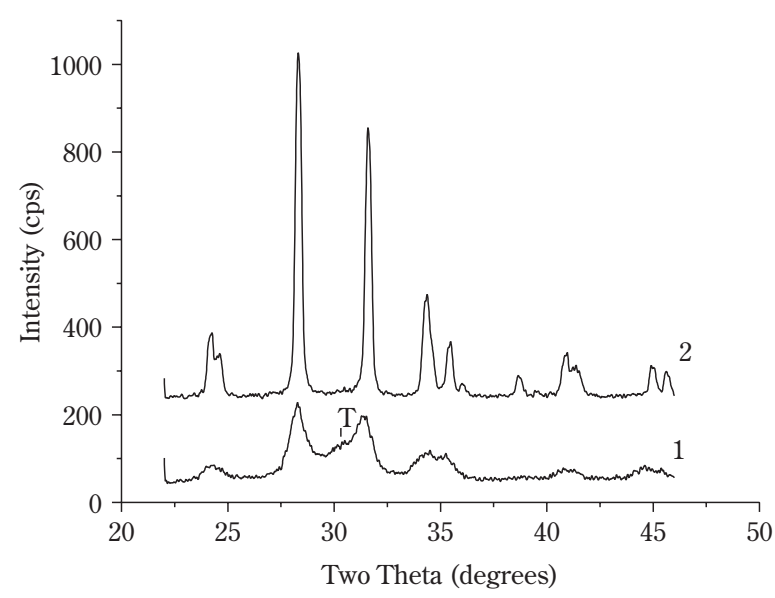

Fig. 10 X-ray pattern of zirconia milled at $20 \mathrm{~g}$ for $10 \mathrm{~min}$ with (1) and without (2) cooling of the shell the growing plasticity of the particles with the rise of temperature, which in turn facilitates their coalescence and the crystallite growth, respectively. Therefore, the threshold size of transformation to tetragonal modification cannot be achieved and the monoclinic phase does not appear.

The contradiction with [26], where the authors were successful in running a complete mechanochemical phase transition is probably a result of the grinding media wear. In the course of intensive grinding with steel balls, fine metal particles will oxidize and the $\mathrm{Fe}^{3+}$ cation integrates into the oxide crystal lattice [30], by analogy to the $\mathrm{Ca}^{2+}$ and $\mathrm{Y}^{3+}$ cations (see below). Thus, the tetragonal phase becomes stabilized independent of the crystallite size. In its turn, the resulting solid solution decomposes at elevated temperatures [30].

Our experiments made under conditions where additives (balls and shell are ceramic) were excluded have demonstrated that even after 10 minutes of grinding (acceleration 20g), the phase transition of the $\mathrm{ZrO}_{2}$ monoclinic modification into the tetragonal one is about $30 \%$. This value remains almost unaffected by the increase of the grinding time up to 1 hour or a rise of the intensity up to $40 \mathrm{~g}$.

Clearly, if the ultrafine tetragonal modification is mechanically treated, then this process will correspond to the conditions described by the left parts of curves 2 and 3 (Figure 1). In this case, even a weak mechanical action should cause the pronounced growth of the crystallite size followed by a transition into the monoclinic phase, as was observed in practice $[25,27]$.

Following our concept, the addition of $\mathrm{Al}$ to $\mathrm{ZrO}_{2}$ should promote the intense grinding of the oxide. By restricting the growth of crystallites, this additive will prevent a reverse phase transition into the monoclinic modification, as shown in Figure 11 (compare with curve 4, Figure 9), even at high temperatures.

Interestingly enough, the additive separating the crystallites from each other can fulfill its function even if it is present only at the grinding stage. In particular, instead of aluminum, we used polyvinyl alcohol that obviously evaporates from the powder long before $800-900^{\circ} \mathrm{C}$. This procedure also prevents transition into the monoclinic phase under heating conditions. Clearly, the growth of crystallites in a nonaggregated powder is possible only if the temperature is higher than the sintering temperature, i.e. if it exceeds $1000^{\circ} \mathrm{C}$.

From the above discussion, it is obvious that it is impossible to obtain fine-dispersed zirconia as well as 


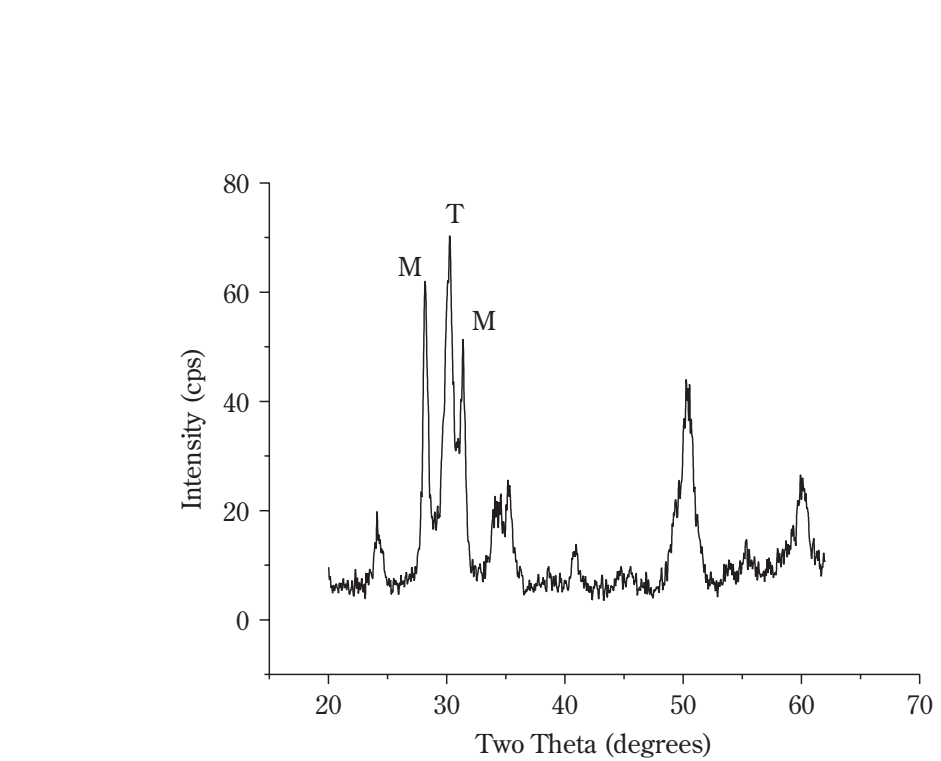

Fig. 11 X-ray pattern of zirconia milled with 15\% $\mathrm{Al}$ additive and annealed at $1000^{\circ} \mathrm{C}$ for 3 hours. (T-tetragonal, M-monoclinic modification.)

other metal oxides, only by intense grinding without additives and specially developed techniques. However, it was possible to make positive use of the same features of the mechanochemical grinding that prevent nanopowders from being obtained.

A problem of great concern in ceramics made of $\mathrm{ZrO}_{2}$ is that the path to obtaining yttrium- or calciumstabilized phases is rather involved. The addition of proper compounds of yttrium or calcium zirconia followed by grinding under conditions favorable for mechanochemical reaction yields stabilized oxide directly in the mill without the need for any solutions.

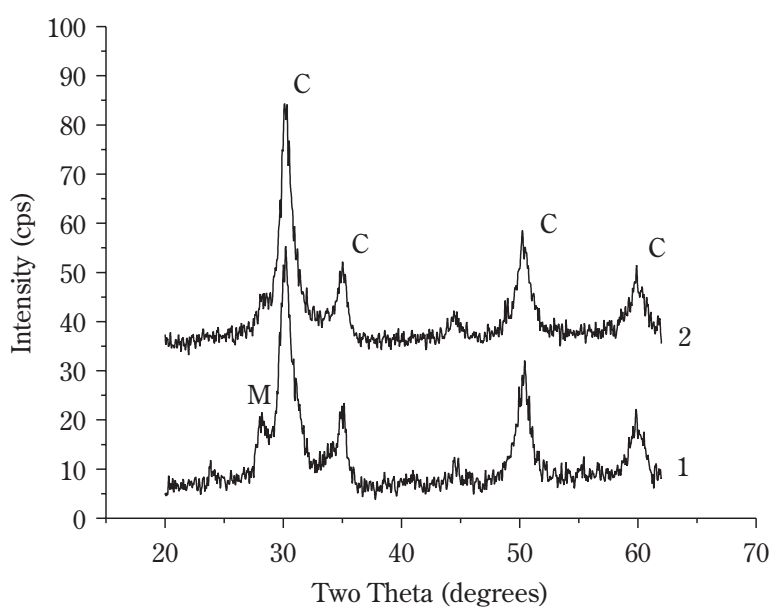

Fig. 12 X-ray patterns of mechanically treated mixtures of $\mathrm{ZrO}_{2}+6 \mathrm{~mol} . \% \mathrm{Y}_{2} \mathrm{O}_{3}$ (2) and $\mathrm{ZrO}_{2}+15 \mathrm{~mol} . \% \mathrm{CaO}$ (1). C cubic phase, $\mathrm{M}-$ monoclinic
In Figure 12, one can see the results of such experiments (see also [31]). The above data cannot provide unambiguous evidence as to whether the tetragonal modification obtained is a result of grinding, where the role of an additive is played by the second oxide, or whether the process advances and we observe the product of a mechanochemical reaction. In either case, whether we obtain a stabilized oxide or only its highly reactive precursor, this product can be directly used in the ceramic production. The sintered samples are a dense $100 \%$ cubic $\mathrm{ZrO}_{2}$.

\section{Conclusions}

From our viewpoint, the results presented together with other experimental data cited in this publication can be successfully interpreted within the scope of a rather rough but very useful scheme. The entirety of processes that occur under intensive mechanical treatment of the powders in a planetary mill, vibratory mill, ball mill, etc. can be described by the counterbalance between reducing the size of the powder particles and crystallites and their agglomeration and coalescence under the action of the external factors of pressure and temperature. By aiming deliberately at this equilibrium by means of preventing or, on the contrary, by facilitating the coalescence of particles, it is possible to obtain nanocomposites or the products of mechanochemical reactions. Making provisions for the subsequent removal of the hindrance (additive), one can obtain quite finely dispersed powders. Moreover, by considering mechanochemical processes with various parameters, including those modified by additives or by the wear of the grinding media, it is possible to influence the general factors responsible for powder contamination, and thus to noticeably increase the purity of the powder.

\section{Literature}

1. Karch, J.; Birringer, R.; Gleiter, H.: "Ceramics ductile at low temperature” Nature, 330, 1987, 556-558.

2. Hahn, H.: "Microstructure and Properties of Nanostructured Oxides.”, Nanostructured Materials, 2, 1993, 251-265.

3. Siegel, R.W.: "Nanostructured Materials - Mind Over Matter”, NanoStructured Materials, 4, 1994, 121-138.

4. Kear, B.H.; Strutt, P.R.: "Nanostructures: The Next Generation of High-Performance Bulk Materials and Coatings”, Kona, 13, 1995, 45-55.

5. Gusev, A.I.: "Nanokristallicheskie materially", Ekaterinburg, 1998

6. Weertman, J.R.; Farkas, D.; Hemker, K.; Kung, H.; 
MdyO 21.; Mitra, R.; Swygenhoven, H.: "Structure and de Mechanical Behavior of Bulk Nanocrystalline MateriWals”, MRS Bulletin, 24, 1999, 44-50.

7. Gutsch, A.; Kramer, M.; Micael, G.; Muhlenberg, H.; Pridohl, M.; Zimmerman, G.: "Gas-Phase Production of Nanoparticles”, KONA, 20, 2002, 24-35.

8. Tsuzuki, T.; McCormick, P.G.: "Synthesis of MetalOxide Nanoparticles by Mechanochemical Processing”, Materials Science Forum, 343-346, 2000, 383-388.

9. Jimbo, G.; Zhao, O.O.; Yokoyama, T.; Taniyama, Y.: "The Grinding Limit and the Negative Grinding Phenomenon", Proc. 2nd World Congress Particle Technology, Kyoto, Japan, 1990, pp.305-312.

10. Bokhonov, B.; Pavlukhin, Yu.; Rykov, A.I.; Paramzin, S.M.; Boldyrev, V.V.: "Crystal growth during mechanical activation of zinc ferrite.”, J. of Mater. Synth. and Process, 1, 1993, 341-346.

11. Alekseenko, V.V.; Karagedov, G.R.: "Interaction of Solids Subjected to Mechanical Stress”, Intern. J. Mechanochem. and Mechanical Alloying, 1, 1994, 1-6.

12. Bokhonov, B.B.; Konstanchuk, I.G.; Boldyrev, V.V.: "Structural and Morphological Changes during the Mechanical Activation of Nano-Size Particles.”, Materials Research Bulletin, 30, 1995, 1277-1284.

13. Alekseenko, V.V.; Karagedov, G.R.: "Interaction of Crystals under Conditions Modelling Mechanical Activation”, Neorganicheskie Materialy, 33, 1997, 817-821.

14. Rusakov, A.A: Rentgenografia Metallov, Moscow, Atomizdat, 1977.

15. Avvakumov E.G.: Mechanical Methods of Chemical Process Activation, Novosibirsk, Nauka, 1986.

16. G.R. Karagedov: "Mechanism of Mechanochemical Synthesis in Oxide Systems”, Proc. 4th Japan-Russia Symp. on Mechanochemistry, Nagoya, 1992, 137-147.

17. Fischmeister, H.F.: "Progress in the Understanding of Ceramic Microstructures and Interfaces since 1976”, Materials Science Research (Ceramic Microstructures '86), 21, 1-14, Plenum press.

18. Sumita S.; Rhine W.E.; Bowen H.K.: "Effects of Organic Dispersants on the Dispersion, Packing, and Sintering of Alumina”, J.Amer.Ceram.Soc., 74, 1991, 2189-2196.
19. Karagedov, G.R.; Lyakhov, N.Z.: "Preparation and Sintering of Nanosized alpha- $\mathrm{Al}_{2} \mathrm{O}_{3}$ powder", NanoStructured Materials, 11, 1999, 559-572.

20. Okada, K.; Kuriki, A.; Hayashi, S.; Yano, T.; Otsuka, N.: "Mechanochemical Effect for Some $\mathrm{Al}_{2} \mathrm{O}_{3}$ Powders by Attrition Milling”, J.Mater.Sci.Letters, 12, 1993, 862864.

21. Gaffet, E.; Bernard, F.; Niepce, J.-C.; Charlot, F.; Gras, C.; Caer, G.: "Some Recent Developments in Mechanical Activation and Mechanosynthesis”, J. Materials Chemistry, 9, 1999, 305-314.

22. McCormic, P.G.: "Application of Mechanical Alloying to Chemical Refining”, Materials Transactions, 36, 1995, 161-169.

23. Takacs, L.: "Reduction of Magnetite by Aluminum: a Displacement Reaction Induced by Mechanical Alloying”, Mater. Letters, 13, 1992, 119-124.

24. Pardavi-Horvath, M.; Takacs, L.: "Iron-Alumina Nanocomposites Prepared by Ball Milling”, IEEE Transactions on Magnetics, 28, 1992, 3186-3188.

25. Bailey, J.E.; Lewis, D.; Librant, Z.M.; Porter, L.J.: "Phase Transformations in Milled Zirconia”, J.Brit.Ceram.Soc., 71, 1972, 25-30.

26. Kuznetsov, P.N.; Zhizhaev, A.M.; Kuznetsova, L.I.: "Mechanochemical synthesis of nanosize metastable zirconium oxide”, RUSS J APPL CHEM ENG TR, 75, 2002, 171-176.

27. Whitney, E.D.: Trans.Fraday Soc., 61, 1965, 1991-1996.

28. Karagedov, G.R.; Ryzhikov, E.A.; Shazkaya, S.S.: "Peculiarities of $\alpha-\mathrm{Al}_{2} \mathrm{O}_{3}$ and $\mathrm{ZrO}_{2}$ Nanogrinding", Chemistry for Sustainable Development, 10, 2002, 89-98.

29. Kwon, Y.-S.; Gerasimov, K.B.; Yoon, S.K.: "Ball Temperatures during Mechanical Alloying in Planetary Mills", J.Alloys and Compounds, 346, 2002, 276-281.

30. Cao, W.; Tan, O.K.; Zhu, W.; Jiang, B.; "Mechanical alloying and thermal decomposition of $\left(\mathrm{ZrO}_{2}\right)(0.8)$ (alpha- $\mathrm{Fe}_{2} \mathrm{O}_{3}$ ) (0.2) powder for gas sensing applications”, J SOLID STATE CHEM., 155, 2000, 320-325.

31. Michel, D.; Faudot, F.; Gaffet, E.; Mazerolles, L.: "Stabilized Zirconias Prepared by Mechanical Alloying”, J.Amer.Ceram.Soc., 76, 1993, 2884-2888. 


\section{Author's short biography}

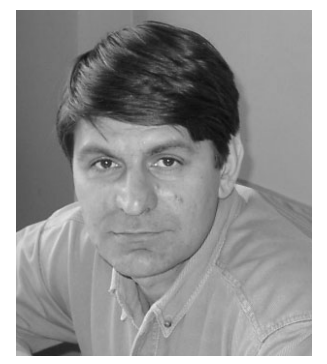

\section{Karagedov Garegin}

Garegin Karagedov graduated from Novosibirsk State University as a chemist in 1977. He received his $\mathrm{PhD}$ (kandidate of science) in physical chemistry in 1986 in Institute of Solid State Chemistry Russian Academy of Sciences. His postdoctoral research included mechanochemical, sol-gel, and radiation-thermal synthesis of superfine powders of multicomponent inorganic oxides. In 1990 he has built up the separate research group aimed at preparation of nanopowders and sintering nanostructured ceramics. His activity in the field resulted in more than 40 publications in peer-reviewed journals.

Garegin Karagedov is currently heading the team for development the technology of advanced uranium oxide fuel rods at Novosibirsk Chemical Concentrates plant.

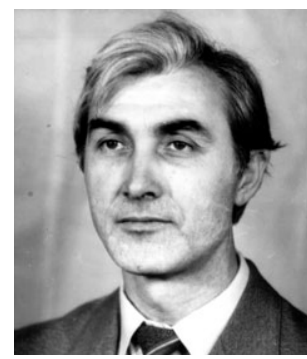

\section{Lyakhov Nikolai}

Nikolai Lyakhov is Director of the Institute of Solid State Chemistry. He graduated from Novosibirsk State University (Physical Faculty) in 1969. He received his $\mathrm{PhD}$ from the Institute of Catalysis in 1976. The main scientific activity is connected with reactivity of solids, mechanochemistry, materials chemistry, synchrotron radiation.

N. Lyakhov is Professor at Novosibirsk State University, corresponding member of Russian Academy of Sci., Member of Asia-Pacific Academy of Materials, President of MRS-Russia, Vice-President of Mendeleev Russian Chemical Society. 